\title{
PSEUDO-RC-INJECTIVE MODULES
}

Mehdi Sadiq Abbas, Mahdi SalehNayef

Department of Mathematics, College of Science, University of Al-Mustansiriyah, Baghdad, Iraq

Department of Mathematics, College of education, University of Al-Mustansiriyah, Baghdad,Iraq

\section{ABSTRACT}

The main purpose of this work is to introduce and study the concept of pseudo-rc-injective module which is a proper generalization of rc-injective and pseudo-injective modules. Numerous properties and characterizations have been obtained. Some known results on pseudo-injective and rc-injective modules generalized to pseudo-rc-injective. Rationally extending modules and semisimple modules have been characterized in terms of pseud-rc-injective modules. We explain the relationships of pseudo-rc-injective with some notions such as Co-Hopfian, directly finite modules.

\section{Indexing terms/Keywords}

Pseudo-injective modules; rc-injective modules; rc-quasi-injective; rationally closedsubmodules;pseudo-rc-injective modules; pseudo-c-injective modules; co-Hopfian modules.

\section{Academic Discipline And Sub-Disciplines}

Mathematic: algebra.

\section{SUBJECT CLASSIFICATION}

16D50, 16D70

\section{Council for Innovative Research}

Peer Review Research Publishing System

JOURNAL OF ADVANCES IN MATHEMATICS

Vol .11, No.4

www.cirjam.com , editorjam@gmail.com 


\section{INTRODUCTION}

Throughout, $R$ represent an associative ring with identity and all $\mathrm{R}$-modules are unitary right modules.

Let $M$ and $N$ be two $R$-modules, $N$ is called pseudo $-M$ - injective if for every submodule $A$ of $M$, any $R$ monomorphism $f: A \rightarrow N$ can be extended to an $R$-homomorphism $\alpha: M \rightarrow N$. An $R$-module $N$ is called pseudo-injective, if it is pseudo $N$-injective. A ring $R$ is said to be pseudo-injective ring, if $R_{R}$ is pseudo-injective module (see [5] and [14]).

A submodule $K$ of an $R$-module $M$ is called rationally closed in $M$ (denoted by $\mathrm{K} \leq_{r c} M$ ) if $N$ has no proper rational extension in $M$ [1]. Clearly, every closed submodule is rationally closed submodule (and hence every direct summand is rationally closed), but the converse may not be true (see [1],[6],[9]).

M. S. Abbas and M. S. Nayef in [3] introduce the concept of rc-injectivity. Let $M_{1}$ and $M_{2}$ be $R$-modules. Then $M_{2}$ is called $M_{1}-r c$-injective if every $R$-homomorphism $f: H \rightarrow M_{2}$, where $H$ is rationally closed submodule of $M_{1}$, can be extended to an $R$-homomorphism $g: M_{1} \rightarrow M_{2}$. An $R$-module $M$ is calledrc-injective, if $M$ is $N$-rc-injective, for every $R$-module $N$. An $R$ module $M$ is called $r c$-quasi-injective or self- $r c$-injective, if $M$ is $M-r c$-injective.

In [15], an $R$ - module $N$ is called pseudo- $M$-c-injective if for any monomorphism from a closed submodule of $M$ to $N$ can be extended to homomorphism from $M$ in to $N$. An $R$-module $M$ is called rationally extending (or RCS-module), if each submodule of $M$ is rational in a direct summand. This is equivalent to saying that every rationally closed submodule of $M$ is direct summand. It is clear that every rationally extending $R$-module is extending [1]. An $R$-module $M$ is said to be Hopfian (Co-Hopfian), if every surjective (injective) endomorphism $f: M \rightarrow M$ is an automorphism [16]. An $R$ - module $M$ is called directly finite if it is not isomorphic to a proper direct summand of $M$ [10]. An $R$-module $M$ is said to be monoform, if each submodule of $M$ is rational [17].

\section{Pseudo-rc-injectiveModules}

We start with the following definition

Definition 2.1Let $M$ and $N$ be two $R$-modules. Then $N$ is pseudo $M$-rationally closed-injective ( briefly pseudo $M$-rcinjective ) if for every rationally closed submodule $H$ of $M$, any $R$-monomorphism $\varphi: H \rightarrow N$ can be extended to an $R-$ homomorphism $\beta: M \rightarrow N$. An $R$-module $N$ is called pseudo-rc-injective, if $N$ is pseudo $N$-rc-injective. Aring $R$ is called self pseudo- rc-injective if it is a pseudo- $R_{R}$-rc-injective.

Remarks 2.2 (1)Every pseudo-injective module is rc-pseudo- injective. The converse may not be true in general, as following example let $M=Z$ as $Z$ - module. Then, clearly $M$ is rc- pseudo-injective, but $Z$ is not pseudo-injective module. This shows that pseudo-rc-injective modules are a proper generalization of pseudo-injective.

(2) Clearly every rc-injective is pseudo-rc-injective. The converse may not be true in general. For example, [7, lemma 2], let $M$ be an $R$-module whose lattice of submodules is
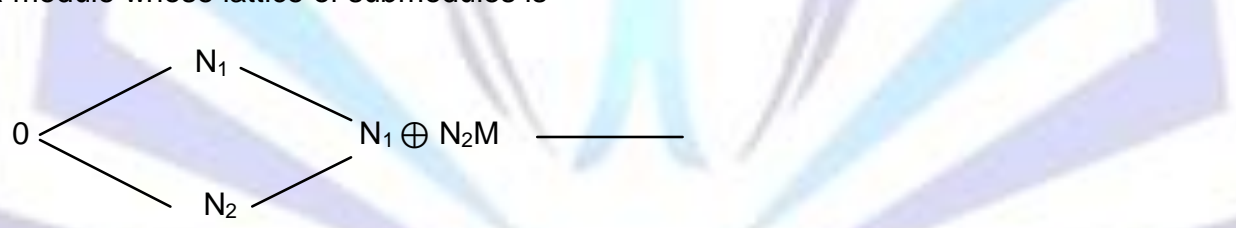

Where $N_{1}$ is not isomorphic to $N_{2}$, and the endomorphism rings of $\mathrm{N}_{\mathrm{i}}$ are isomorphic to $Z / 2 Z$ where $\mathrm{i}=1,2$. $\mathrm{S}$. Jain and $\mathrm{S}$. Singh in [7] are show that, $M$ is pseudo-injective (and hence by (1), $M$ is pseudo-rc-injective) which is not rc-quasiinjective, since $N_{1} \oplus N_{2}$ is rationally closed submodule of $M$ and the natural projection of $N_{1} \oplus N_{2}$ onto $N i(\mathrm{i}=1,2)$ can not be extended to an $R$-endomorphism of $M$,[7]. Therefore, $M$ is not rc-injective module. This shows that pseud-rc-injective modules are a proper generalization of rc-injective modules.

(3) Obviously, every pseudo- $M$-rc-injective is pseudo $M$-c-injective. The converse is not true in general. For example, consider the two $Z$ - module $M=Z / 9 Z$ and $N=Z / 3 Z$ it is clear that $N$ is pseudo $M$ - $c$ - injective but $N$ is not pseudo - $M$ rc- injective. This shows that pseud-rc-injective modules are stronger than of rc-injective modules.

Proof: Let $H=<3>$, clearly $H$ is rationally closed submodule of $M$, and define $\alpha: H \rightarrow N$ by $\alpha(0)=0, \alpha(3)=1$, $\alpha(6)=2$. Obvious, $\alpha$ is $Z$ - monomorphism. Now, suppose that $N$ is pseudo $M$-rc-injective then there is $\beta: M \rightarrow N$ and $\beta(1)=n$ for some $n \in N$. Hence $\beta(3)=3 \beta(1)=3 n$ and hence $3 n=\beta(3)=\alpha(3)=1$, implies $3 n=1$, a contradiction, this shows that , $N$ is not pseudo $M$-rc-injective.

(4) For a non-singular $R$ - module $M$. If $N$ is pseudo $M$-c-injective then $N$ is pseudo $M$-rc-injective.

(5) Every monoform $R$-module is pseudo-rc-injective.

(6) An $R$-isomorphic module to pseudo-rc-injective is pseudo-rc- injective. 
So, by above we obtain the following implications for modules.

$$
\begin{aligned}
& \text { Injective } \Rightarrow \text { quasi-injective } \Rightarrow \text { pseudo-injective } \Rightarrow \text { pseudo-rc-injective } \Rightarrow \text { pseudo-c-injective. } \\
& \text { Rc-injective } \Rightarrow \text { rc-quasi-injective } \Rightarrow \text { pseudo-rc-injective } \Rightarrow \text { pseudo-c-injective. }
\end{aligned}
$$

In the following result we show that, for a uniform $R$-module the concepts of the rc-injective modules and pseudorc-injective are equivalents.

Theorem 2.3 Let $M$ be uniform $R$ - module. $M$ is a rc-injective if and only if $M$ is a pseudo-rc-injective module.

Proof: $\Longrightarrow)$ Obviously.

$(\Leftarrow) \quad$ Suppose that $M$ is a pseudo-rc- injective, let $K$ be rationally closed submodule of $M$ and $\alpha: K \rightarrow M$ be $R$ homomorphism. Since $M$ is uniform module, either $\alpha$ or $I_{K}-\alpha$ is a $R$-monomorphism. First, if $\alpha$ is $R$-monomorphism, then by pseudo-rc-injectivity of $M$, there exists $R$-homomorphism $g: M \rightarrow M$ such that $g \circ i_{K}=\alpha$.Finally, if $I_{K}-\alpha$ is $R$ monomorphism, then by pseudo-rc-injectivity of $M$, there exists $g: M \rightarrow M$ such that $g \circ i_{K}=I_{K}-\alpha$ hence $I_{K}-g=\alpha$. Therefor $M$ is rc- injective.

Proposition 2.4 Let $N_{1}$ and $N_{2}$ be two $R$-modules and $N=N_{1} \oplus N_{2}$. Then $N_{2}$ is pseudo $N_{1}$-rc-injective if and only if for every (rationally closed) submodule $A$ of $N$ such that $A \cap N_{2}=0$ and $\pi_{1}(A)$ rationally closed submodule of $N_{1}$ (where $\pi_{1}$ is a projection map from $N$ onto $N_{1}$ ), there exists a submodule $A^{\prime}$ of $N$ such that $A \leq A^{\prime}$ and $N=A^{\prime} \oplus N_{2}$.

Proof: Similar to proving [3, proposition (2.3)].

Some general properties of pseudo- $r c$-injectivity are given in the following results.

Proposition 2.5Let $M$ and $N_{i}(i \in I)$ be $R$-modules. Then $\prod_{i \in I} N_{i}$ is pseudo $M$ - $r c$-injective if and only if $N_{i}$ is pseudo $M$ $r c$-injective, for every $i \in I$.

Proof: Follows from the definition and injections and projections associated with the direct product.

The following corollary is immediately from proposition (2.5).

Corollary 2.6Let $M$ and $N_{i}$ be $R$-modules where $i \in I$ and $I$ is finite index set, if $\oplus_{i=1}^{n} N_{i}$ is pseudo $M$-rc-injective, $\forall i \in I$ , then $N_{i}$ is pseudo- $M$-rc-injective. In particular every direct summand of pseudo-rc-injective $R$-module is pseudo-rcinjective.

Proposition 2.7 Let $M$ and $N$ be $R$-modules. If $M$ is pseudo $N$-rc-injective, then $M$ is pseudo $A$-rc-injective for every rationally closed submodule $A$ of $N$.

Proof: Let $A \leq_{r c} N$ and let $K \leq_{r c} A, f: K \rightarrow N$ be $R$-monomorphism. Then, by [2, Lemma (3.2)] we obtain, ( $K \leq_{r c} N$, hence by pseudo $N$-rc-injectivity of $M$, there exists a $R$-homomorphism $h: N \rightarrow M$ such that $h \circ i_{A} \circ i_{K}=f$ where $i_{K}: K \rightarrow A$ and $i_{A}: A \rightarrow N$ are inclusion maps. Let $\varphi=h \circ i_{A}$. Clearly, $\varphi$ is $R$-homomorphism, and $\varphi=h \circ i_{k}=h \circ i_{A} \circ i_{k}=f$ Then $\varphi$ is extends $f$. Therefore, $M$ is $A$-rc- injective.

In [15] was proved the following: Suppose that $R$ is a commutative domain. Let $c$ be a non-zero non-unit element of $R$. The right $R$-module $R \oplus(R / x R)$ is not pseudo-c-injective. From this result and remark (2.2)(3), we conclude the following proposition for pseudo-rc-injective modules.

Proposition 2.8 For a commutative domain $R$. Let $x$ be a non-zero non-unit element of $R$. The $R$-module $R \oplus(R / x R)$ is not pseudo $r c$-injective.

Now, we investigate more properties of pseudo rc-injectivity.

The $R$-module $M_{1}$ and $M_{2}$ are relatively (mutually) pseudo-rc- injective if $M_{i}$ is pseudo $M_{j}$-rc - injective for every $i, j \in$ $\{1,2\}, i \neq j$.

The following result is generalization of [5, Theorem (2.2)].

Theorem 2.9 If $M \oplus N$ is a pseudo-rc-injective module, then $M$ and $N$ are mutually rc-injective.

Proof: Suppose that $M \oplus N$ is a pseudo-rc-injective module. Let $B$ be a rationally closed submodule of $N$ and $\alpha: B \rightarrow M$ be an $R$-homomorphism. Define $\varphi: B \rightarrow M \oplus N$ by $\varphi(b)=(\alpha(b), b)$ for all, $b \in B$, it is clear that $\varphi$ is an $R$-monomorphism, . Since $N$ is isomorphic to a direct summand of $M \oplus N$, then (by remark (2.2)(3)) and proposition(2.7), we have $M \oplus N$ is pseudo-rc $N-$ injective, thus, there exists an $R$-homomorphism $f: N \rightarrow M \oplus N$ such that $\varphi=f \circ i_{B}$ where $i_{B}: B \rightarrow N$ be the inclusion map. Let 
$\pi_{1}=M \oplus N \rightarrow M$ be natural projection of $M \oplus N$ onto $M$. We have $\pi_{1} \circ \varphi=\pi_{1} \circ f \circ i_{B}$ and hence $\alpha=\pi_{1} \circ f \circ i_{B}$, thus $\pi_{1} \circ$ $f: N \rightarrow M$ is $R$-homomorphism extending $\alpha$. This show that $M$ is $N$-rc-injective. As same way we can prove that $N$ is $M$-rcinjective.

Corollary 2.10 If $\oplus_{i \in I} M_{i}$ is a pseudo -rc - injective, then $M_{i}$ is a $M_{j}$-rc-injective for all distinct $i, j \in I$.

Corollary 2.11 For any positive integer $\mathrm{n} \geq 2$, if $M^{n}$ is pseudo rc- injective, then $M$ is rc-quasi-injective.

The following example shows that the direct sum of two pseudo-rc-injective is not pseudo-rc-injective in general. For a prim $\mathrm{p}$, let $M_{1}=Z$ and $M_{2}=Z / p Z$, be a right $Z$-modules . Since $M_{1}$, and $M_{2}$ are monoform then, $M_{1}$, and $M_{2}$ are pseudo-rcinjective. But, by proposition (2.8), we have $M_{1} \oplus M_{2}$ is not pseudo-rc -injective module. injective.

Now, we consider the sufficient condition for a direct sum of two pseudo- rc- injective modules to be pseudo -rc-

Theorem 2.12 The direct sum of any two pseudo-rc-injective modules is pseudo-rc- injective if and only if every pseudo -rc- injective module is injective.

Proof:Let $M$ be a pseudo-rc-injective module, and $E(M)$ its injective hull of $M$. By hypothesis, we have $M \oplus E(M)$ is pseudo-rc-injective. Let $i_{M}: M \rightarrow M \oplus E(M)$ be a natural injective map then there exists an R-homomorphism $\alpha: M \oplus$ $E(M) \rightarrow M \oplus E(M)$ such that $i_{M}=\alpha \circ i_{E} \circ i$, where $i: M \rightarrow E(M)$ is inclusion map and $i_{E}: E(M) \rightarrow M \oplus E(M)$ is injective map. Thus, $I_{M}=\pi_{M} \circ i_{M}=\pi_{M} \circ \alpha \circ i_{E} \circ i$, where $I_{M}$ is the identity of $M$ and $\pi_{M}$ is a projection map from $M \oplus E(M)$ onto $M$. Therefore $I_{M}=g \circ i$, where $g=\pi_{M} \circ \alpha \circ i_{E}$. Thus by [8, Corollary (3.4.10)], we obtain $E(M)=M \oplus k e r g$. Since $M \cap$ $\operatorname{kerg}=0$ and $M \leq_{e} E(M)$ lead to $\operatorname{kerg}=0$ and hence $M=E(M)$. This shows that $M$ is injective module. The other direction is obvious. $\square$

Recall that an $R$-module $M$ is a multiplication if, each submodule of $M$ has the form $I M$ for some ideal $I$ of $R$ [9].

Proposition 2.13 Every rationally closed submodule of multiplication pseudo-rc-injective $R$-module is pseudo-rcinjective.

Proof: Let $A$ be a rationally closed submodule of a rationally closed submodule $H$ of $M$ and let $f: A \rightarrow H$ be an $R$ monomorphism. Since $H$ is a rationally closed of $M$. It follows that by [2, Lemma (3.2), $A$ is also a rationally closed submodule of $M$. Since $M$ is pseudo-rc-injective, then there exist an $R$-homomorphism $\varphi: M \rightarrow M$ that extends $f$. Since $\mathrm{M}$ is multiplication module, we have $H=M I$ for some ideal $\mathrm{I}$ of $R$. Thus $\left.\varphi\right|_{H}=\varphi(H)=\varphi(M I)=\varphi(M) I \leq M I=H$. This show that $H$ is pseudo-rc-injective.

In the following part we give characterizations of known $R$-modules in terms of pseudo-rc-injectivity.

We start with the following results which are given a characterization of rationally extending modules. Firstly, the following lemma is needed.

Lemma 2.14 Let $A$ be rationally closed submodule of $R$-module $M$. If $A$ is pseudo $M$-rc-injective, then $A$ is a direct summand of $M$.

Proof: Since $\mathrm{A}$ is a pseudo $M$-rc-injective $R$-module, there exists an $R$-homomorphism $f: M \rightarrow A$. That extends The identity $I: A \rightarrow A$. Hence by [8, Corollary (3.4.10), $M=A \oplus \operatorname{kerf}$, so that $A$ is a direct summand of $M$.

Proposition 2.15 An $R$-module $M$ is rationally extending if and only if every $R$-module is pseudo $M$ - $r c$-injective.

Proof: $\Rightarrow)$. It is similarly to prove [3, proposition (2.4).

$(\Longleftarrow)$. Follow from lemma (2.14).

Note that, by proposition (2.15), every rationally extending $R$-module is pseudo-rc-injective. But the converse is not true in general. As in the following example: consider the $Z$-module $M=Z / p^{2} Z$ where $p$ is prime number. It is clear that, $M$ is pseudo- $r c$-injective (in fact, $M$ is rc-injective). Obviously, $A=\langle P\rangle$ is rationally closed submodule of $M$ but $A$ is not direct summand of $M$. Thus $M$ is not rationally extending.

Theorem 2.16 For an $R$-module $M$, the following statements are equivalent:

(1) $M$ is rationally extending;

(2) Every $R$-module is an $M$-rc-injective;

(3) Every $R$-module is pseudo $M$-rc-injective;

(4) Every rationally closed submodule of $M$ is an $M$-rc-injective;

(5) Every rationally closed submodule of $M$ is a pseudo $M$-rc-injective.

Proof:(1) $\Leftrightarrow$ (2) Follows from [3, proposition (2. 4). 
(2) $\Rightarrow$ (4). Clear.

$(4) \Rightarrow(1)$. It is follows from lemma (2.14).

Now, (1) $\Rightarrow$ (3). It is follows from proposition (2.15).

(3) $\Rightarrow(5)$. It is obvious.

$(5) \Rightarrow(1)$. It is follows from lemma (2.14).

An $R$ - module $M$ is directly finite if and only if $f \circ g=I_{M}$ implies that $g \circ f=I_{M} \quad$ for all $f, g \in$ End (M) [10, proposition (1.25)]. The $Z$-module $Z$ is directly finite, but it is not co-Hopfian. In the following proposition we show that the co-Hopfian and directly finite $\mathrm{R}$-modules are equivalent under pseudo-rc-injective property.

Proposition 2.17A pseudo-rc-injective $R$-module $M$ is directly finite if and only if it is co-Hopfian.

Proof: Let $\varphi$ be an injective map belong to $E n d(M)$ and I is identity $R$-homomorpism from $M$ to $M$. By pseudo-rc-injectivity of $M$, there exists an $R$-homomorphism $\beta: M \rightarrow M$ such that $\beta \circ \varphi=I_{M}$. Since $M$ is directly finite, we have $\varphi \circ \beta=I_{M}$ which is shows that $\varphi$ is an $R$-automorphism. Therefore, $M$ is co-Hopfian. The other direction it is clear.

The following corollary is immediately from proposition (2.17).

Corollary 2.18An rc-injective $R$-module $M$ is directly finite if and only if it is Co-Hopfian. $\square$

Since every indecomposable module is directly finite then by proposition (2.17), we obtain the following corollary.

Corollary 2.19 If $M$ is an indecomposable pseudo-rc-injective module then $M$ is a Co-Hopfian.

In [33] was proved that every Hopfian $R$-module is directly finite. Thus the following result follows from proposition (2.17).

Corollary 2.20 If $M$ is a pseudo-rc-injective and Hopfian $R$ - module .Then $M$ is a Co-Hopfian. $\square$

For any an $R$-module $M$ we consider the following definition.

Definition 2.21 An $R$-module $M$ said to be complete rationally closed module (briefly $\boldsymbol{C R C}$ module), if each submodule of $M$ is a rationally closed. It is clear that every semisimple module is $C R C$ module, but the converse is not true in general.

For example $Z_{4}$ as $Z$-module is $C R C$ module, but not semisimple since $<2>$ is not direct summand of $Z_{4}$.

An $R$-module $M$ is said to be satisfies $\left(\mathrm{C}_{2}\right)$-condition, if for each submodule of $M$ which is isomorphic to a direct summand of $M$, then it is a direct summand of $M[10]$.Recall that an $R$-module $M$ is said to satisfy the generalized $\mathrm{C}_{2}$-condition (or $\mathrm{GC}_{2}$ ) if, any $N \leq M$ and $N \cong M, N$ is a summand of $M[18]$.

The following result is a generalization of [5, Theorem (2.6)]

Proposition 2.22Every pseudo-rc-injective $\mathrm{CRC}$ module satisfies $\mathrm{C}_{2}$ (and hence $\mathrm{GC}_{2}$ ).

Proof: Let $M$ be a pseudo-rc- injective CRC module, let $H \leq M$ and $K \leq M$ such that $H$ is isomorphic to $K$ with $H \leq{ }_{d} M$. Since $M$ is a pseudo-rc- injective then by corollary(2.6), we obtain $H$ is a pseudo- $M$-rc- injective. But $H \cong K$ thus, by remark (2.2)(9), $K$ is a pseudo $M$-rc-injective. By assumption, we have $K$ is rationally closed sub module of $M$. Thus, by Lemma (2.14), we get $K \leq_{d} M$.Hence $M$ satisfies $C_{2}$. The last fact follows easily.

Although the Z-module $M=Z$ is a pseudo-rc-injective, but it is not satisfies $\mathrm{C}_{2}$, since there is a submodule $H=n Z$ (where $(n \geq 2)$ ) of which is isomorphic to $M$ but it is not a direct summand in $M$. This shows that the CRC property of the module in proposition (2.22) cannot be dropped.

In [4], an $R$-module $M$ is called direct-injective, if given any direct summand $K$ of $M$, an injection map $j_{K}: K \rightarrow M$ and every R-monomorphism $\alpha: K \rightarrow M$, there is an R-endomorphism $\beta$ of $M$ such that $\beta \alpha=j_{K}$.

In [11, Theorem (7.13)], it was proved that, an $R$-module $M$ is a direct-injective if and only if $M$ is satisfies $\left(\mathrm{C}_{2}\right)$-condition. Thus by proposition (2.22) we can conclude the following result.

Proposition 2.23 Every pseudo-rc-injective CRC module is direct-injective.

In [13, p.32], recall that a right $R$-module $M$ is called divisible, if for each $m \in M$ and for each $r \in R$ which is not left zerodivisor, there exist $m^{\prime} \in M$ such that $m=m^{\prime} r$. In [4] was proved that every direct-injective $R$-module is divisible. Thus we have the following corollary which follows from proposition (2.23).

Corollary 2.24Every pseudo-rc-injective CRC module is divisible. 
Recall that an $R$-module $M$ is self-similar if, every submodule of $M$ is isomorphic to $M$ [12].The $Z$-module $Z$ is both selfsimilar and pseudo-rc-injective module but it is not semisimple and CRC module.Also, $Z_{4}$ as $Z$-module is pseudo-rcinjective CRC module but it is not self-similar module.Note that from above examples the concepts CRC-modules and selfsimilar modules are completely different.

In the following result we show that the pseudo-rc-injective and semisimple $R$-modules are equivalent under self-similar CRC modules.

Theorem 2.25Let $M$ is a self-similar CRC module. Then the following statements are equivalent:

(i) $\quad M$ is semisimple module;

(ii) $\quad$ Mis pseudo-rc-injective.

Proof: (i) $\Rightarrow$ (ii). Clear.

(ii) $\Rightarrow$ (i). Let $K$ be any submodule of $M$, then by self-similarity of $M$, we have $K$ is isomorphic to $M$. Since $M$ is pseudorc-injective CRC module thus, by proposition (2.22), $M$ satisfy $\mathrm{GC}_{2}$-condition. So, $K$ is a direct summand of $M$. therefore, $M$ is semisimple module.

\section{REFERENCES}

[1]. Abbas, M. S. and Ahmed, M. A. (2011), Rationally Extending and Strongly Quasi-Monoform Modules, AlMustansiriya J. Sci. Vol. 22, No 3,pp. 31-38.

[2]. Mehdi S. Abbas and Mahdi S. Nayef, (2015), RATIONALLY INJECTIVE MODULES, Journal of Advances in Mathematics Vol.10, No.5, 3479-3485.

[3]. Mehdi S. Abbas and Mahdi S. Nayef, (2015), M-RC-Injective and RC-Quasi-injective Modules, International J. of Math. Sci. Vol.35, Issue. 2,1772-1779.

[4]. Chang-woo Han and Su-JeongChol, (1995), Generalizations of the quasi-injective modules, Comm. Korean Math. Soc. No.4, 10, 811-813.

[5]. Dinh, H. Q. (2005), A note on Pseudo-injective modules, comm. Algebra, 33, 361-369.

[6]. Goodearl, K. R. (1976), Ring Theory, Nonsingular Rings and Modules, Marcel Dekker. Inc. New York.

[7]. Jain, S. K. and Singh, S. (1975), Quasi-injective and Pseudo-injective modules. Canada. Math.Bull.18, 359-366.

[8]. Kasch, F., (1982), Modules and Rings, Academic Press Inc. London (English Translation).

[9]. Lam,T. Y., (1999), Lectures on Modules and Rings. GTN 189, Springer Verlag, New York.

[10]. Mohamed, S. H. and Muller, B. J. (1990), Continuous and Discrete modules, London Math.Soc. Lecture note Series 14, Cambridge Univ. Press.

[11]. Nicholson,W. K. andYousif,M. F. (2003), Quasi-Frobenius Rings, Cambridge Univ. Press.

[12]. Rodrigues,V. S. Sant'Ana, A. A. (2009), A not on a problem due to Zelmanowitz, Algebra and Discrete Mathematics, No.3, 85-93.

[13]. Sharpe, N. D. W. andVamos, P. (1972), Injective Modules, Cambridge Univ. Press.

[14]. Singh, S. and Jain,S. K. (1967), On pseudo-injective modules and self pseudo-injective rings, J. Math. Sci. 2, $23-31$.

[15]. T. Sitthiwirattham, S. Bauprasist and S. Asawasamirt, (2012), On Generalizations of Pseudo-injectivity, Int. Journal of Math. Analysis, Vol. 6, no. 12, 555-562.

[16]. Varadarajan,K. (1992), Hopfian and Co-Hopfian objects. PublicacionsMathematiques, Vol. 36, 293-317.

[17]. Zelmanowits,J. M. (1986), Representation of rings with faithful polyform modules, Comm. Algebra, 14(6),1141-1169.

[18]. Zhou, Y. (2002), Rings in which certain right ideals are direct summands of annihilators, J. Aust. Math. Soc. 73, 335346. 